\title{
Winterhardiness of some winter wheat (Triticum aestivum), rye (Secale cereale), triticale ( $x$ Triticosecale) and winter barley (Hordeum vulgare) cultivars tested at six locations in Finland
}

\author{
LEENA HÖMMÖ and SEPPO PULLI
}

Номмӧ, L. \& РuцLi, S. 1993. Winterhardiness of some winter wheat (Triticum aestivum), rye (Secale cereale), triticale ( $\mathrm{x}$ Triticosecale) and winter barley (Hordeum vulgare) cultivars tested at six locations in Finland. Agric. Sci. Finl. 2: 311-327. (Agric. Res. Centre of Finland, Inst. Plant Breed., FIN-31600 Jokioinen, Finland.)

\begin{abstract}
The winterhardiness of 24 winter wheat, 13 rye, 5 triticale and 11 winter barley varieties of different origins was tested at six locations in Finland in 1989-1992. The survival ability of the cultivars, their resistance to snow mould (Microdochium nivale) and the correlations between these traits and the growth habit and growth stage were determined.

The trials were grouped on the basis of variety ranking, and the differences between the varieties within each group were studied by the analysis of variance. Statistically highly significant differences between varieties were found in all cases. The wintering conditions during the trials were very variable, and this brought about differences in the ranking of cultivars in different trials. In most cases the genotypic-environmental interactions could be explained by the different genetic systems controlling the tolerance to various winter stresses and changes in their intensity.
\end{abstract}

Key words: cold tolerance, frost resistance, Microdochium nivale, snow mould

\section{Introduction}

Winter cereals are grown mainly in temperate regions of the world. The ability of species to acclimate (harden) during autumn to survive the extreme conditions in winter determines their northern limit of distribution. During the hardening period, numerous changes take place in the plant (LEVITT 1972). The rates and extent of these changes depend upon both the genotype of the plant and the prevailing environmental conditions (FOWLER and GUSTA 1977). According to FOWLER and LIMIN (1987), the maximum coldhardiness of the most winter hardy cultivars of barley (Hordeum vulgare $\mathrm{L}$.$) , triticale ( \mathrm{x}$ Triticosecale Wittmack), wheat (Triticum aestivum L.) and rye (Secale ce- reale $\mathrm{L}$.) is $-19^{\circ} \mathrm{C},-24^{\circ} \mathrm{C},-25^{\circ} \mathrm{C}$ and $-34^{\circ} \mathrm{C}$, respectively. This order of coldhardiness is also reflected in their commercial cultivation areas in the Nordic countries: Denmark, Sweden, Norway, Finland and Iceland. Winter barley is grown almost only in Denmark $(175,000$ ha in 1993) and in Southern Sweden $(10,700$ ha in 1992). The winter triticale also meets its northern limit in Denmark $(1,000$ ha in 1993) and in Southern Sweden (19,400 ha in 1992). Winter wheat is grown up to the southern parts of Finland (Fig. 1), and the southern and costal areas of Norway. The area of winter wheat cultivation was in Denmark 610,000 ha (1993), in Sweden 235,000 ha (1992) and in Norway 33,000 ha (1993). In Finland, during the last five years the winter wheat area has varied between 46,400 ha 
(1991) and 12,300 ha (1992), depending on both the climatic factors and policy. The northern limit of distribution of rye is in central Finland (Fig.1). The area of rye cultivation was in Denmark 70,000 ha (1993), in Sweden 34,100 ha (1992) in Norway 2,000 ha (1993) and in Finland it has ranged between 84,800 ha (1990) and 10,600 ha (1992) during the last five years. In Iceland, only some experiments have been made on winter cereals, but there is no commercial cultivation.

Since the spring forms of species avoid the possible economic losses caused by the winter damages, spring barley is cultivated in all the Nordic countries but Denmark, and spring wheat exceeds winter wheat in Finland and in Norway. The higher yield level, the better drought resistance in early summer and early harvesting in autumn have, however, made the winter forms very important also in the northern limits of distribution. This has urged the development of better agronomic practices for cultivating winter cereals (ANDERSEN 1992, ANDERSSON 1986, BENGTSSON 1986, BRUEHL 1982, FOWLER et al. 1976, GUDLEIFSSON 1986, GUSTA 1986, HÄNNINEN and JAMALAINEN 1968, HETHERINGTON et al. 1990, JAMALAINEN 1974, NisSINEN 1986, Olvång 1992, POHJaKallio et al. 1962, POMEROY and ANDREWS 1989, PULLI 1986, SMITH 1986, 1987 and URVAS 1986) and also to find some new resources of winterhardiness to be utilized in developing new, more winter resistant cultivars (ANDREWS et al. 1986, BRULE-BABEL and FOWLER 1987, DVORAK and FOWLER 1978, FOWLER et al. 1977, HeNSLEIGH et al. 1992, JENKINS 1963, LAZAR et al. 1988, LimIN et al. 1985, LIMIN and FOWLER 1984, 1986, 1988, 1991, VEISZ and RAJKI 1987). Although numerous sophisticated tests have been developed to measure the hardening ability and resistance of breeding material against different winter stresses, field trials are, in most cases, still used to give the ultimate evaluation of the winter hardiness of the studied lines or cultivars.

In the present study, the winterhardiness of winter wheat, rye, triticale and winter barley varieties was tested in field trials established at six locations in Finland (Fig.1) in 1989-1992. On the basis of these results the tested cultivars were arranged according to their resistance against different winter stresses, and the suitability of the experimental sites for screening the winterhardiness of tested species was discussed.

\section{Material and methods}

The field trials comprised 24 ( 22 in 1989-90) winter wheat, 13 (11 in 1989-90) winter rye, 5 winter triticale and 11 (10 in 1989-90) winter barley varieties. The varieties and their origins are listed in Tables 1 and 7.

The field trials were carried out at four locations: Anjalankoski $\left(60^{\circ} 43^{\prime} \mathrm{N}, 26^{\circ} 48^{\prime} \mathrm{E}\right)$, Pälkäne $\left(61^{\circ} 20^{\prime} \mathrm{N}, 24^{\circ} 13^{\prime} \mathrm{E}\right)$, Laukaa $\left(62^{\circ} 20^{\prime} \mathrm{N}, 26^{\circ} 10^{\prime} \mathrm{E}\right)$ and Sotkamo $\left(64^{\circ} 06^{\prime} \mathrm{N}, 28^{\circ} 20^{\prime} \mathrm{E}\right)$ in 1989-1990. In 1990-1991 and 1991-1992 trials were established also at Jokioinen $\left(60^{\circ} 49^{\prime} \mathrm{N}, 23^{\circ} 30^{\prime} \mathrm{E}\right)$ and Mietoinen $\left(60^{\circ} 38^{\prime} \mathrm{N}, 21^{\circ} 51^{\prime} \mathrm{E}\right)$ (Fig. 1$)$.

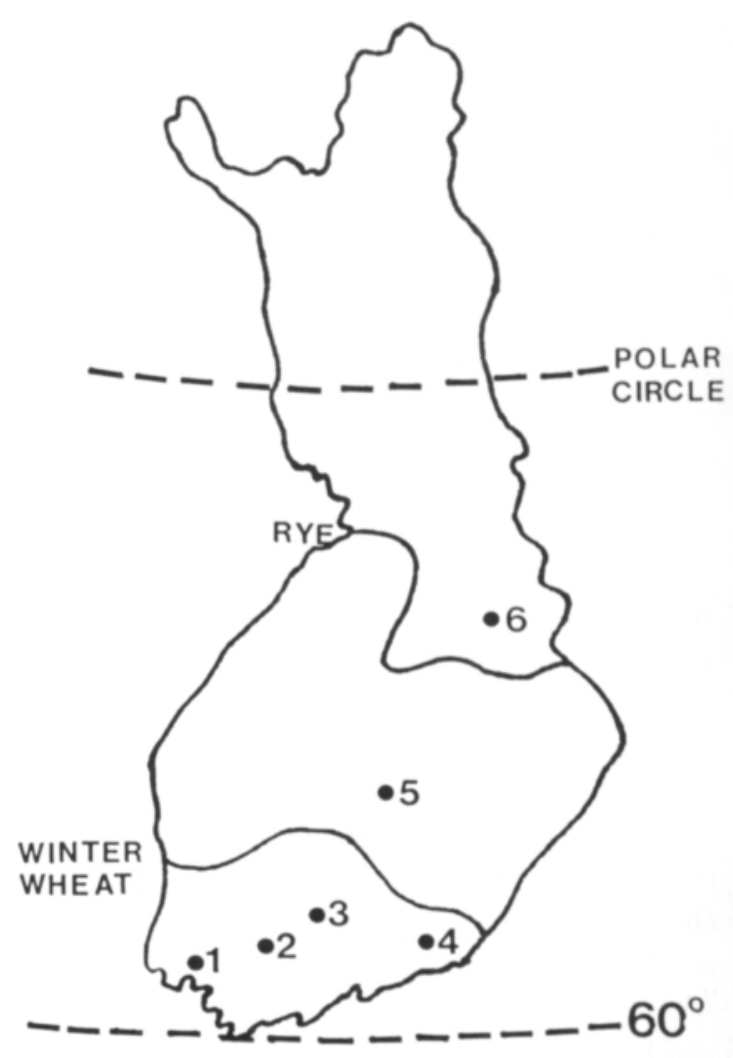

Fig. 1. Locations of the field trials. $1=$ Mietoinen, $2=$ Jokioinen, 3 = Pälkäne, 4 = Anjalankoski, 5 = Laukaa and 6 = Sotkamo. The northern limits of winter wheat and rye cultivation are also shown on the map. 
The trials were completely randomized with four replicates and the cultivars were sown in $1 \mathrm{~m}$ rows. The trials were sown in August and they were fertilized in autumn and in spring according to the normal practice of the experimental stations.

In autumn, the growth habit of varieties was determined using a scoring system of 1 (prostrate) to 5 (erect). In autumn 1990 at Jokioinen and Mietoinen, 10 plants of each variety were selected randomly from the first replicate and the number of leaves (growth stage) and the height of the plants were measured.

The winter survival rate of varieties was determined by counting the plants both in the autumn and in the spring soon after the snow had melted. At the same time, damage to the plants caused by snow mould (Microdochium nivale (Fr.) Samu \& Hall) was rated using the scoring system of 0 (totally undamaged) to 10 (dead).

The climatic data concerning the temperatures and the snow cover at the experimental sites was obtained from the Monthly Reports of the Meteorological Institute of Finland.

Differences in winter survival abilities between experimental varieties were studied using the analysis of variance and Tukey's studentized range test. The arcsin modification of data was done before the analysis.

The correlations between winter survival of varieties, snow mould resistance, growth habit, number of leaves and height of plants were determined using the correlation analysis.

The field trials were part of the Inter-Nordic "Winterhardiness" Project. The same winter wheat, rye, triticale and winter barley varieties were tested in the same years in all the Nordic countries at 17 locations. The test locations were in Denmark Tystofte, Ris $\emptyset$, Abed, Pajberg and Sejet; in Sweden: Svalöv and Uppsala; in Norway: Apelsvoll, Kvithamar and Vågønes and in Iceland: Möðruvellir. The Inter-Nordic field trial results collected by Dr Kurt Hjortsholm were kindly offered for use in this study. The data was divided into two groups on the basis of the assumed main stress factor, snow or frost, and the winter survival of varieties was determined within each group. The correlations between the combined Inter-Nordic field test results and the overwintering results in Finland were determined.

\section{Results}

The level of general survival of each studied species varied greatly depending on the year and experimental site (Fig. 2). The variation was greater among winter wheat and winter barley varieties than among the rye and triticale varieties (Fig. 2). Winter rye was the most winter hardy species, winter wheat was somewhat hardier than winter triticale, and winter barley was inferior in this respect. Since wheat variety 'Vitus' behaved more like spring wheat in it's ability to harden during autumn and therefore had very low winter survival (Table 1), it was excluded from further testing.

\section{Winter wheat}

The field survival of winter wheat varieties at different locations in 1989-1992 is shown in Table 1. The analysis of variance indicates that in some trials the differences between varieties were not significant (Table 1). In some cases this was due to a very mild winter period (Mietoinen 1992 and Jokioinen 1992), which caused only slight damage to the plants. Ice encasement at Anjalankoski 1991 resulted in very clear but uneven injury to the cultivars, but the differences between varieties were obscured by the great variation between replicates.

The ranges of variation within each trial are shown in Figure 2. Very significant positive correlations were found between all the trials in which there was a wide variation between varieties. This was obvious for Jokioinen 1991, Pälkäne 1990, Anjalankoski 1991, Laukaa 1990 and 1991 and Sotkamo 1990, 1991 and 1992. All these trials were characterized by cold winter with rather long period of snow cover, and they were pooled to form group 1 . The results of this group correlated well also with all the results from Inter-Nordic trials and with the total results from Finnish trials (Table 2).

The group 2 trials were Mietoinen 1991, Pälkäne 1991, Anjalankoski 1990 and 1992 and Laukaa 

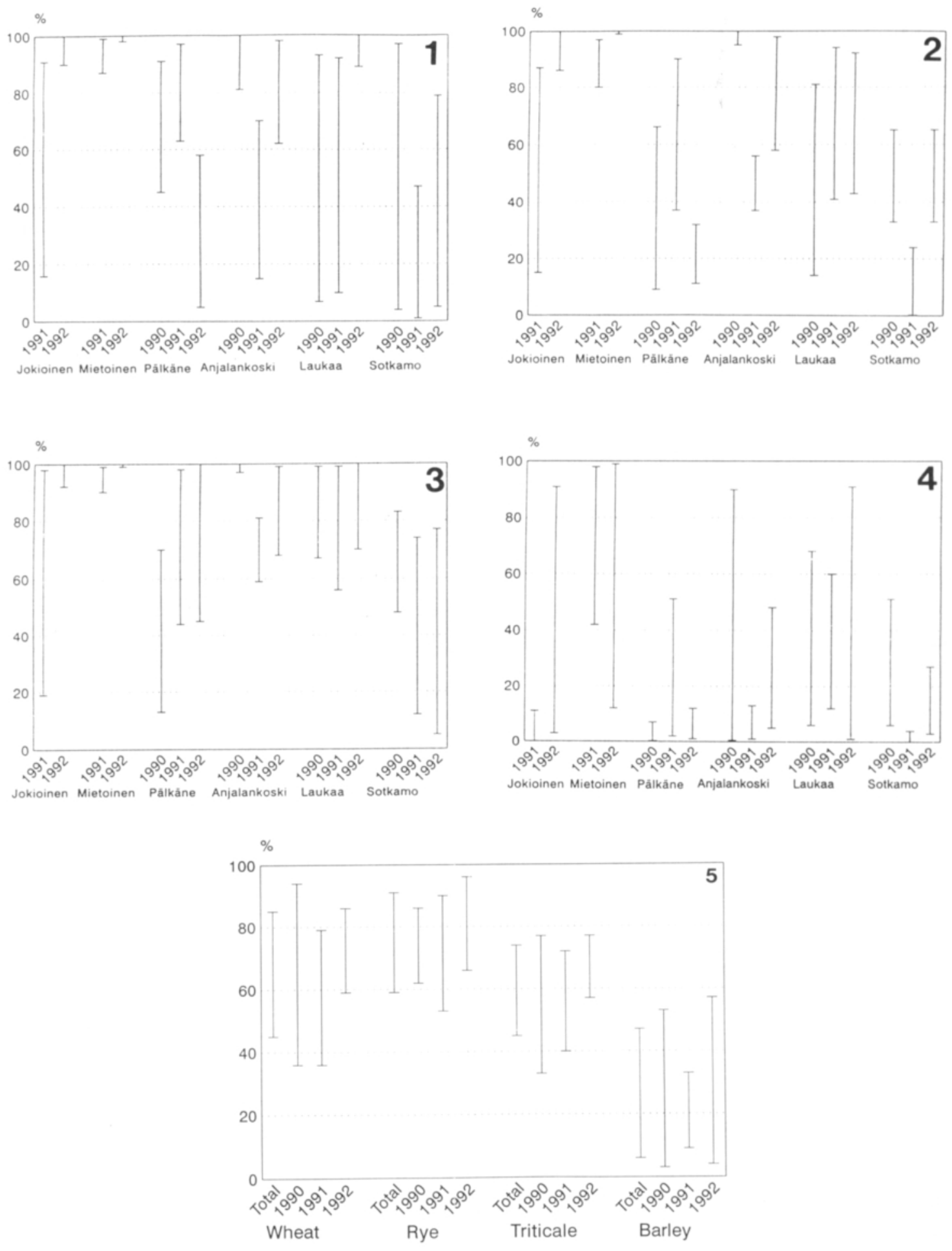

Fig. 2. Variation in winter survival ability of winter wheat (1), triticale (2), rye (3) and winter barley (4) varieties, and total variation of each species in each of the experimental years (5). Winter wheat variety 'Vitus' was excluded because it is similar in hardiness to spring wheats. 


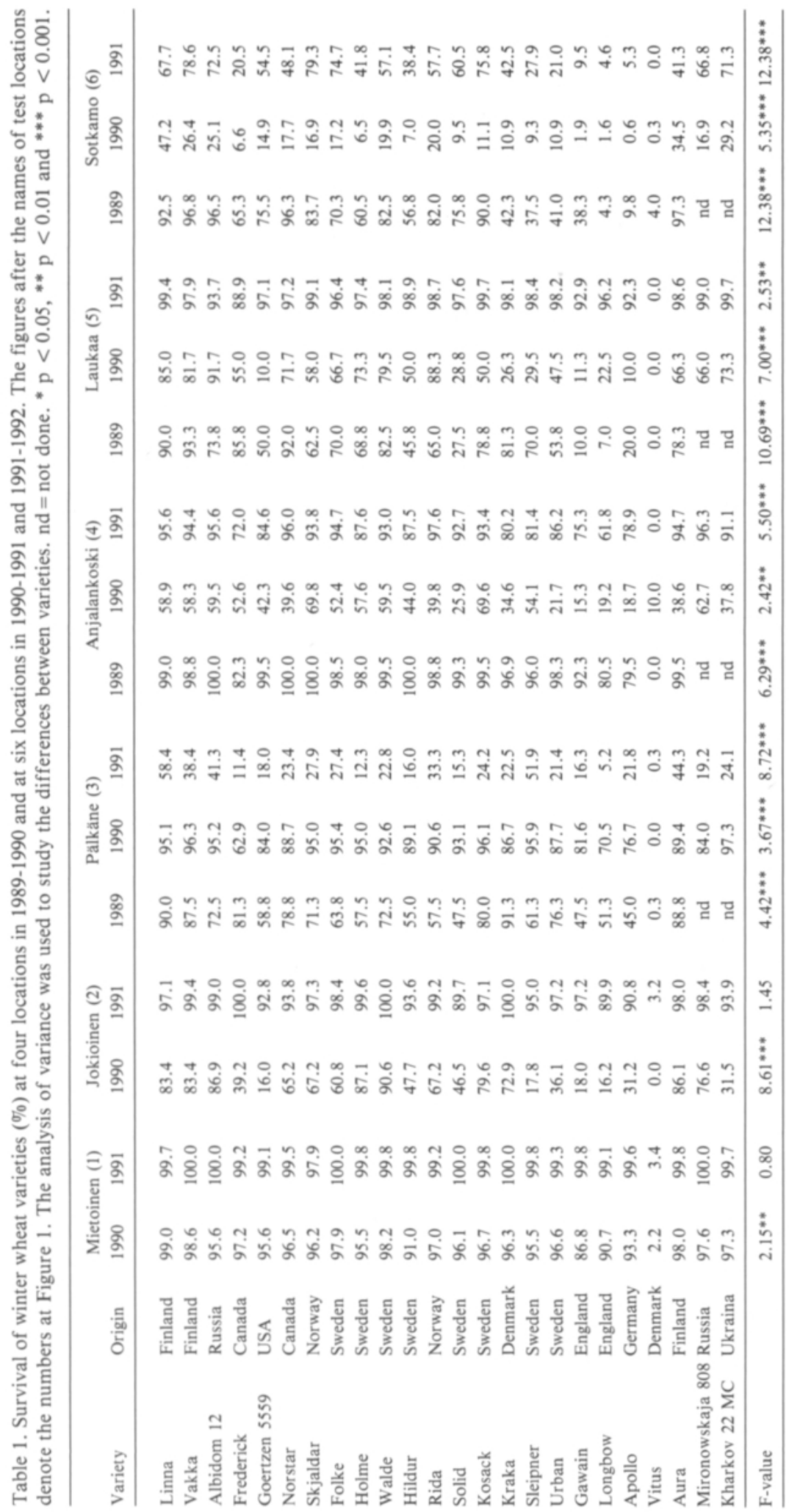


Table 2. Correlations between winter survival in the winter wheat, rye and winter barley trials, and mean winter survival in the Inter-Nordic (Total frost, Total snow, Total I-N) and Finnish trials in 1989-1992 and means of all trials. ${ }^{*} \mathrm{p}<0.05$, ** $\mathrm{p}<0.01, * * * \mathrm{p}<0.001$.

\begin{tabular}{|c|c|c|c|c|c|c|c|c|}
\hline \multicolumn{2}{|c|}{ Winter wheat } & \multirow{2}{*}{$\begin{array}{c}\begin{array}{c}\text { Total } \\
\text { frost }\end{array} \\
0.63^{* * *}\end{array}$} & \multirow{2}{*}{$\begin{array}{c}\begin{array}{c}\text { Total } \\
\text { snow }\end{array} \\
0.82^{* * *}\end{array}$} & \multirow{2}{*}{$\begin{array}{c}\begin{array}{c}\text { Total } \\
\text { I-N }\end{array} \\
0.74^{* * *}\end{array}$} & \multirow{2}{*}{$\begin{array}{c}\begin{array}{c}\text { Finland } \\
1990\end{array} \\
0.79^{* * *}\end{array}$} & \multirow{2}{*}{$\begin{array}{c}\begin{array}{c}\text { Finland } \\
1991\end{array} \\
0.73^{* * *}\end{array}$} & \multirow{2}{*}{$\begin{array}{c}\begin{array}{c}\text { Finland } \\
1992\end{array} \\
0.71^{* * *}\end{array}$} & \multirow{2}{*}{$\begin{array}{c}\begin{array}{c}\text { Finland } \\
\text { total }\end{array} \\
0.77^{* * *}\end{array}$} \\
\hline Mietoinen & 1991 & & & & & & & \\
\hline & 1992 & 0.11 & 0.15 & 0.15 & 0.12 & 0.16 & 0.17 & 0.15 \\
\hline \multirow[t]{2}{*}{ Jokioinen } & 1991 & $0.83^{* * *}$ & $0.86^{* * *}$ & $0.86^{* * *}$ & $0.78^{* * *}$ & $0.89^{* * *}$ & $0.71^{* * *}$ & $0.83^{* * *}$ \\
\hline & 1992 & $0.54^{* *}$ & $0.61^{* *}$ & $0.59^{* *}$ & $0.63^{* *}$ & $0.61^{* *}$ & $0.41^{*}$ & $0.59^{* *}$ \\
\hline \multirow[t]{3}{*}{ Pälkäne } & 1990 & $0.65^{* * *}$ & $0.76^{* * *}$ & $0.71^{* * *}$ & $0.82^{* * *}$ & $0.62^{* *}$ & $0.55^{* *}$ & $0.71^{* * *}$ \\
\hline & 1991 & $0.55^{* *}$ & $0.66^{* * *}$ & $0.63^{* *}$ & $0.54^{*}$ & $0.64^{* * *}$ & $0.80^{* * *}$ & $0.67^{* * *}$ \\
\hline & 1992 & $0.62^{* *}$ & $0.62^{* *}$ & $0.64 * *$ & $0.54^{* *}$ & $0.54^{* *}$ & $0.68^{* * *}$ & $0.60^{* *}$ \\
\hline \multirow[t]{3}{*}{ Anjalank. } & 1990 & $0.60^{* * *}$ & $0.70^{* * *}$ & $0.67^{* * *}$ & $0.68^{* * *}$ & $0.64^{* *}$ & $0.79^{* * *}$ & $0.73^{* * *}$ \\
\hline & 1991 & $0.69 * * *$ & $0.76^{* * *}$ & $0.75^{* * *}$ & $0.72^{* * *}$ & $0.79 * * *$ & $0.69^{* * *}$ & $0.77^{* * *}$ \\
\hline & 1992 & $0.77^{* * *}$ & $0.82^{* * *}$ & $0.81 * * *$ & $0.75^{* * *}$ & $0.81^{* * *}$ & $0.91 * * *$ & $0.85^{* * *}$ \\
\hline \multirow[t]{3}{*}{ Laukaa } & 1990 & $0.76^{* * *}$ & $0.89^{* * *}$ & $0.84^{* * *}$ & $0.93 * * *$ & $0.81^{* * *}$ & $0.71^{* * *}$ & $0.87^{* * *}$ \\
\hline & 1991 & $0.85^{* * *}$ & $0.83^{* * *}$ & $0.85^{* * *}$ & $0.75^{* * *}$ & $0.91 * * *$ & $0.70^{* * *}$ & $0.84^{* * *}$ \\
\hline & 1992 & 0.36 & $0.44^{*}$ & 0.42 & 0.37 & $0.44^{*}$ & $0.59 * *$ & $0.47^{*}$ \\
\hline \multirow[t]{3}{*}{ Sotkamo } & 1990 & $0.87^{* * *}$ & $0.89^{* * *}$ & $0.89^{* * *}$ & $0.90^{* * *}$ & $0.83^{* * *}$ & $0.86^{* * *}$ & $0.90^{* * *}$ \\
\hline & 1991 & $0.86^{* * *}$ & $0.82^{* * *}$ & $0.85^{* * *}$ & $0.76^{* * *}$ & $0.75^{* * *}$ & $0.79 * * *$ & $0.79^{* * *}$ \\
\hline & 1992 & $0.78^{* * *}$ & $0.82^{* * *}$ & $0.83^{* * *}$ & $0.75^{* * *}$ & $0.80^{* * *}$ & $0.91 * * *$ & $0.84^{* * *}$ \\
\hline \multicolumn{9}{|l|}{ Winter rye } \\
\hline \multirow[t]{2}{*}{ Mietoinen } & 1991 & 0.28 & $0.84^{* * *}$ & $0.70^{*}$ & $0.85^{* * *}$ & $0.63^{*}$ & $0.72^{* *}$ & $0.71^{* *}$ \\
\hline & 1992 & -0.13 & 0.44 & 0.28 & 0.35 & 0.32 & 0.52 & 0.42 \\
\hline \multirow[t]{2}{*}{ Jokioinen } & 1991 & $0.84^{* * *}$ & $0.82^{* * *}$ & $0.91^{* * *}$ & $0.71^{*}$ & $0.93^{* * *}$ & $0.86^{* * *}$ & $0.89^{* * *}$ \\
\hline & 1992 & -0.15 & 0.54 & 0.33 & 0.56 & 0.47 & 0.56 & 0.55 \\
\hline \multirow[t]{3}{*}{ Pälkãne } & 1990 & $0.70^{*}$ & $0.89^{* * *}$ & $0.90^{* * *}$ & $0.89 * * *$ & $0.90^{* * *}$ & $0.86^{* * *}$ & $0.91^{* * *}$ \\
\hline & 1991 & 0.55 & $0.93^{* * *}$ & $0.87^{* * *}$ & $0.90^{* * *}$ & $0.82 * * *$ & $0.86^{* * *}$ & $0.86^{* * *}$ \\
\hline & 1992 & $0.89^{* * *}$ & $0.84^{* * * *}$ & $0.93^{* * *}$ & $0.81^{* *}$ & $0.91 * * *$ & $0.86 * * *$ & $0.90^{* * *}$ \\
\hline \multirow[t]{3}{*}{ Anjalank. } & 1990 & -0.12 & 0.45 & 0.26 & 0.52 & 0.21 & 0.39 & 0.35 \\
\hline & 1991 & -0.41 & -0.18 & -0.30 & -0.05 & 0.17 & 0.24 & 0.22 \\
\hline & 1992 & 0.51 & $0.77^{* *}$ & $0.74^{* *}$ & $0.72^{* *}$ & $0.68^{* *}$ & $0.70^{* *}$ & $0.71^{* *}$ \\
\hline \multirow[t]{3}{*}{ Laukaa } & 1990 & -0.06 & 0.57 & 0.38 & $0.64^{*}$ & 0.33 & 0.50 & 0.48 \\
\hline & 1991 & $0.81^{* *}$ & $0.70^{*}$ & $0.79^{* *}$ & $0.79^{* *}$ & $0.77^{* *}$ & $0.63^{*}$ & $0.75^{* *}$ \\
\hline & 1992 & 0.20 & $0.73^{* *}$ & $0.60^{*}$ & $0.69^{*}$ & $0.64^{*}$ & $0.77^{* *}$ & $0.73^{* *}$ \\
\hline Sotkamo & 1990 & $0.65^{*}$ & $0.59^{*}$ & $0.64^{*}$ & $0.68^{*}$ & $0.66^{*}$ & 0.40 & $0.60^{*}$ \\
\hline & 1991 & $0.64^{*}$ & $0.90^{* * *}$ & $0.88^{* * *}$ & $0.76^{* * *}$ & $0.77^{* *}$ & $0.69^{* *}$ & $0.73^{* *}$ \\
\hline & 1992 & 0.44 & $0.83^{* * *}$ & $0.76^{* *}$ & $0.61^{*}$ & $0.80^{* * *}$ & $0.90^{* * *}$ & $0.83^{* * *}$ \\
\hline Winter bar & & & & & & & & \\
\hline Mietoinen & 1991 & $0.67^{*}$ & $0.83^{* * *}$ & $0.81^{* *}$ & 0.54 & $0.83^{* * *}$ & $0.80^{* *}$ & $0.80^{* *}$ \\
\hline & 1992 & $0.61^{*}$ & $0.79 * *$ & $0.77^{* *}$ & 0.50 & $0.75^{* *}$ & $0.84^{* * *}$ & $0.78^{* *}$ \\
\hline Jokioinen & 1991 & 0.52 & $0.61^{*}$ & 0.58 & 0.54 & $0.65^{*}$ & 0.55 & $0.62^{*}$ \\
\hline & 1992 & $0.68^{*}$ & $0.85^{* * *}$ & $0.83^{* *}$ & 0.60 & $0.83^{* * *}$ & $0.94^{* * *}$ & $0.87^{* * *}$ \\
\hline Pălkäne & 1990 & $0.84^{* * *}$ & $0.68^{*}$ & $0.74^{* *}$ & $0.78^{* *}$ & 0.50 & 0.57 & $0.67^{*}$ \\
\hline & 1991 & 0.57 & $0.61^{*}$ & $0.62^{*}$ & 0.45 & $0.80^{* *}$ & $0.61^{*}$ & $0.66^{*}$ \\
\hline & 1992 & $0.61^{*}$ & $0.70^{*}$ & $0.70^{*}$ & 0.53 & $0.70^{*}$ & $0.75^{* *}$ & $0.71^{* *}$ \\
\hline Anjalank. & 1990 & $0.88^{* * *}$ & $0.88^{* * *}$ & $0.87^{* * *}$ & $0.97^{* * *}$ & $0.63^{*}$ & $0.72^{*}$ & $0.84^{* *}$ \\
\hline & 1991 & $0.80^{* *}$ & $0.86^{* * *}$ & $0.84^{* *}$ & $0.87^{* * *}$ & $0.65^{*}$ & 0.47 & $0.67^{*}$ \\
\hline & 1992 & 0.23 & 0.48 & 0.43 & 0.19 & $0.73^{* *}$ & $0.60^{*}$ & 0.55 \\
\hline Laukaa & 1990 & $0.78^{* *}$ & $0.78^{* *}$ & $0.77^{* *}$ & $0.88^{* * *}$ & 0.58 & $0.63^{*}$ & $0.75^{* *}$ \\
\hline & 1991 & 0.49 & 0.60 & 0.60 & 0.49 & $0.73^{* *}$ & $0.63^{*}$ & $0.66^{*}$ \\
\hline & 1992 & $0.82 * *$ & $0.84^{* *}$ & $0.82^{* *}$ & $0.94^{* * *}$ & 0.54 & $0.72^{* *}$ & $0.79^{* *}$ \\
\hline Sotkamo & 1990 & $0.83^{* *}$ & $0.84^{* *}$ & $0.83^{* *}$ & $0.89 * * *$ & $0.62^{*}$ & $0.74^{* *}$ & $0.81^{* *}$ \\
\hline & 1991 & 0.41 & 0.57 & 0.51 & 0.47 & $0.59^{*}$ & 0.34 & 0.49 \\
\hline & 1992 & $0.83^{* *}$ & $0.76^{* *}$ & $0.79^{* *}$ & $0.80^{* *}$ & 0.54 & $0.69^{*}$ & $0.70^{*}$ \\
\hline
\end{tabular}

Winter wheat $\mathrm{n}=21$ in Inter-Nordic trials and in 1990 trials, otherwise $\mathrm{n}=23$ rye $\mathrm{n}=11$ or $\mathrm{n}=13$ and winter barley $\mathrm{n}=10$ or $\mathrm{n}=11$, respectively. 
1992. All these trials were characterized by a relatively mild winter. The cold periods were quite short, and the minimum temperatures during the cold season were moderate. In most trials, some damage was caused by snow mould, but on average the survival rate of all the experimental varieties was high, and the variation between varieties was small. A significant positive correlation was found between the individual trials, and in most cases the results of these trials correlated also well with the total results of the Inter-Nordic trials and with the totals of the Finnish trials, but the level of correlation was not so high as in group 1. In both cases, the individual trials correlated better with the InterNordic trials when the stress factor was snow mould (Table 2). The analysis of variance showed significant differences between varieties in both groups. As expected the years and experimental sites differed from each other also very significantly (Table 3). Tukey's Studentized Range test was used to group the varieties on the basis of their winterhardiness (Table 4). In both groups, varieties with high and low winterhardiness could be identified, but the varieties with moderate winterhardiness could only be identified in severe winter conditions. On the basis of these results and the total means of winter survival (Table 4) the most winter hardy varieties were 'Linna', 'Vakka', 'Kosack', 'Albidom 12' and 'Aura', and those least winter hardy were 'Gawain', 'Apollo' and 'Longbow'. Three varieties were excluded from the analysis. Two varieties, 'Mironowskaja 808' and 'Kharkov 22 MC' were not included in the 1990 trials and were therefore excluded from the analysis. These two varieties are both considered to be moderately winter hardy. Since 'Vitus' is similar in hardiness to spring wheats, it was also excluded from the analysis.

The negative but not significant correlation between survival percentage and growth habit, and the highly significant positive correlation between amount of snow mould damage and erect growth habit of winter wheat varieties indicates that prostrate growth habit might be more beneficial for the winter survival of winter wheat (Table 5). There was a highly significant positive correlation between survival rate and size (both growth stage and
Table 3. Analysis of variance for the survival rate of winter wheat in groups 1 and 2, rye in groups 1 and 2, triticale in groups 1 and 2 and winter barley in groups 1,2 and 3 .

${ }^{*} \mathrm{p}<0.05,{ }^{* *} \mathrm{p}<0.01,{ }^{* * *} \mathrm{p}<0.001$.

\begin{tabular}{|c|c|c|c|}
\hline Source of variation & DF & MS & F-value \\
\hline \multicolumn{4}{|l|}{ Winter wheat } \\
\hline \multicolumn{4}{|l|}{ Group 1.} \\
\hline Variety & 20 & 1.70 & $24.29 * * *$ \\
\hline Location & 4 & 1.90 & $27.14^{* * *}$ \\
\hline Year & 2 & 6.06 & $86.57^{* * *}$ \\
\hline Error & 616 & 0.07 & \\
\hline \multicolumn{4}{|l|}{ Group 2.} \\
\hline Variety & 20 & 0.19 & $9.50^{* * *}$ \\
\hline Location & 3 & 0.46 & $23.00 * * *$ \\
\hline Year & 1 & 1.87 & $93.50^{* * *}$ \\
\hline Error & 395 & 0.02 & \\
\hline \multicolumn{4}{|l|}{ Winter rye } \\
\hline \multicolumn{4}{|l|}{ Group 1.} \\
\hline Variety & 12 & 1.31 & $26.20^{* * *}$ \\
\hline Location & 4 & 5.59 & $111.80^{* * *}$ \\
\hline Year & 2 & 4.33 & $86.60^{* * *}$ \\
\hline Error & 383 & 0.05 & \\
\hline \multicolumn{4}{|l|}{ Group 2 . } \\
\hline Variety & 12 & 0.14 & $7.00^{* * *}$ \\
\hline Location & 3 & 0.75 & $37.50^{* * *}$ \\
\hline Year & 2 & 0.40 & $20.00^{* * *}$ \\
\hline Error & 276 & 0.02 & \\
\hline
\end{tabular}

\section{Winter triticale}

Group 1 .

Variety

Location

Year

Error

0.53

$17.67 * * *$

$225.00^{* * *}$

$109.33^{* * *}$

3.28

0.03

Group 2.

Variety

Location

0.72

0.69

$10.29 * * *$

$9.86 * * *$

Error

0.07

Winter barley

Group 1,

Variety

Location

Year

Error

$21.00 * * *$

$39.75 * * *$

$32.75^{* * *}$

Group 2.

Variety

Location

Year

Error

1.59

1.31

0.04

Group 3.

Variety

Location

Year

0.21

0.90

0.59

0.01

$21.00^{* * *}$

$90.00 * * *$

$59.00 * * *$

Error

0.21

$21.00 * * *$

0.90

$90.00^{* * *}$

$59.00^{* * *}$ 
Table 4. Winter survival of winter wheat, rye and winter barley varieties. Means are total means of field trials in 1989-1992 at six locations. The grouping of varieties is based on Tukey's Studentized Range test. ni $=$ not included.

\begin{tabular}{|c|c|c|c|c|}
\hline Winter wheat & Mean & Grouping & & Grouping \\
\hline Group 1 & & & Group 2 & \\
\hline Vakka & 83.5 & $\mathrm{a}$ & Linna & a \\
\hline Linna & 85.2 & $\mathrm{a}$ & Kosack & $a b$ \\
\hline Kosack & 79.0 & $a b c$ & Folke & $a b$ \\
\hline Albidom 12 & 81.3 & $a b c$ & Vakka & $a b$ \\
\hline Aura & 79.4 & $a b c$ & Skjaldar & $a b$ \\
\hline Walde & 77.8 & abcd & Rida & $a b$ \\
\hline Norstar & 77.0 & abcd & Aura & $a b$ \\
\hline Skjaldar & 76.3 & abcd & Walde & $a b$ \\
\hline Folke & 74.1 & bcde & Norstar & $a b$ \\
\hline Rida & 74.3 & bcde & Albidom 12 & $a b$ \\
\hline Holme & 71.1 & bcdef & Solid & $a b$ \\
\hline Kraka & 67.6 & bcdefg & Holme & $a b$ \\
\hline Goertzen 5559 & 63.1 & bcdefg & Sleipner & $a b$ \\
\hline Solid & 62.8 & bcdefg & Hildur & $a b$ \\
\hline Frederick & 64.1 & bcdefg & Urban & $a b c$ \\
\hline Hildur & 64.3 & bcdefg & Goertzen 5559 & $a b c$ \\
\hline Sleipner & 64.0 & cdefg & Kraka & $\mathrm{bc}$ \\
\hline Urban & 62.1 & defg & Gawain & $\mathrm{cd}$ \\
\hline Gawain & 49.6 & h & Apollo & d \\
\hline Apollo & 48.3 & h & Frederick & d \\
\hline Longbow & 45.0 & $\mathrm{~h}$ & Longbow & d \\
\hline Vitus & $1.4 \mathrm{ni}$ & & & \\
\hline Mironovsk. 808 & $73.7 \mathrm{ni}$ & & & \\
\hline Kharkov & $71.3 \mathrm{ni}$ & & & \\
\hline \multicolumn{5}{|l|}{ Rye } \\
\hline Group 1 & & & Group 2 & \\
\hline Jussi & 90.5 & $a b c$ & Jussi & $a b c$ \\
\hline Voima & 89.0 & $a b c$ & Anna & $a b c$ \\
\hline Anna & 87.0 & abcd & Norderåstetra & $a b c$ \\
\hline Norderåstetra & 84.7 & abcde & Talovskaja 12 & abcd \\
\hline Talovskaja 12 & 82.8 & bcde & Voima & abcd \\
\hline Vàgonảs höstråg & 75.6 & cdef & Petkus ii & abcde \\
\hline Prima & 71.4 & efgh & Prima & abcde \\
\hline Danko & 71.8 & fghi & Dominator & abcde \\
\hline Epos & 68.3 & fghij & Danko & bcde \\
\hline Dominator & 71.2 & fghij & Kungs ii & cde \\
\hline Kungs ii & 67.3 & fghij & Musketeer & cdef \\
\hline Petkus ii & 69.0 & ghij & Epos & cdef \\
\hline Musketeer & 59.5 & hij & Vàgonäs höstråg & ef \\
\hline \multicolumn{5}{|l|}{ Winter barley } \\
\hline Group 1 & & & Group 3 & \\
\hline Borwina & 46.7 & $\mathrm{a}$ & WB $158-25$ & $a b$ \\
\hline Andrea & 38.5 & $\mathrm{~b}$ & Andrea & $a b$ \\
\hline Frost & 37.9 & b & Borwina & $a b c$ \\
\hline Marinka & 32.9 & $\mathrm{~cd}$ & Marinka & $a b c$ \\
\hline Lady & 28.2 & $\mathrm{~cd}$ & Frost & $a b c$ \\
\hline Trixi & 30.0 & cd & Trixi & $a b c$ \\
\hline OAC Acton & 24.7 & $\mathrm{~cd}$ & Finesse & $a b c$ \\
\hline Finesse & 29.7 & $\mathrm{~cd}$ & Lady & $a b c$ \\
\hline Igri & 24.2 & $\mathrm{~cd}$ & OAC Acton & $a b c$ \\
\hline WB 158-25 & 26.0 & cde & Igri & bc \\
\hline Maris Otter & 5.9 & de & Maris Otter & $\mathrm{d}$ \\
\hline
\end{tabular}


Table 5. Correlations between winter survival of winter wheat, rye and triticale varieties, and snow mould damage, growth habit, growth stage (= number of leaves) and height of plants. ${ }^{*} \mathrm{p}<0.05,{ }^{* *} \mathrm{p}<0.01$ and ${ }^{* * *} \mathrm{p}<0.001$.

\begin{tabular}{|c|c|c|c|c|}
\hline & Snow mould & Growth habit & Growth stage & Plant height \\
\hline \multicolumn{5}{|l|}{ Winter wheat: } \\
\hline Survival $\%$ & $\begin{array}{r}-0.51^{* * *} \\
\mathrm{n}=898\end{array}$ & $\begin{array}{l}-0.24 \\
\mathrm{n}=40\end{array}$ & $\begin{array}{l}0.56^{* * *} \\
\mathrm{n}=40\end{array}$ & $\begin{array}{l}0.54 * * * \\
\mathrm{n}=40\end{array}$ \\
\hline Snow mould & & $\begin{array}{l}0.53^{* * *} \\
\mathrm{n}=40\end{array}$ & $\begin{array}{l}-0.06 \\
n=40\end{array}$ & $\begin{array}{l}-0.01 \\
n=40\end{array}$ \\
\hline \multicolumn{5}{|l|}{ Winter rye: } \\
\hline Survival $\%$ & $\begin{array}{r}-0.21^{* * * *} \\
\mathrm{n}=560\end{array}$ & $\begin{array}{l}0.51^{* *} \\
\mathrm{n}=25\end{array}$ & $\begin{array}{l}0.17 \\
\mathrm{n}=25\end{array}$ & $\begin{array}{l}0.43^{*} \\
\mathrm{n}=25\end{array}$ \\
\hline Snow mould & & $\begin{array}{l}-0.07 \\
\mathrm{n}=25\end{array}$ & $\begin{array}{l}-0.34 \\
\mathrm{n}=25\end{array}$ & $\begin{array}{l}-0.42^{*} \\
\mathrm{n}=25\end{array}$ \\
\hline \multicolumn{5}{|l|}{ Triticale: } \\
\hline Survival $\%$ & $\begin{array}{r}-0.60^{* * *} \\
\mathrm{n}=210\end{array}$ & $\begin{array}{l}-0.23 \\
\mathrm{n}=10\end{array}$ & $\begin{array}{l}0.51 \\
\mathrm{n}=10\end{array}$ & $\begin{array}{l}0.31 \\
\mathrm{n}=10\end{array}$ \\
\hline Snow mould & & $\begin{array}{l}0.27 \\
\mathrm{n}=10\end{array}$ & $\begin{array}{l}-0.29 \\
\mathrm{n}=10\end{array}$ & $\begin{array}{l}-0.25 \\
\mathrm{n}=10\end{array}$ \\
\hline
\end{tabular}

height of the plants) of the wheat varieties (Table 5). Thus the robust plants which synthesize more reserves during the autumn survive better.

The amount of damage to varieties in different trials caused by snow mould (Microdochium nivale) is shown in Table 6. On the basis of analysis of variance the differences in snow mould resistance between winter wheat varieties were significant only in five trials out of twelve (Table 6). On the basis of the total means of the two years, the most snow mould resistant winter wheat varieties were 'Linna', 'Folke', 'Sleipner', 'Aura' and 'Vakka' and those most susceptible were 'Longbow', 'Frederick', 'Apollo', 'Gawain' and 'Goertzen 5559 '. There was a highly significant negative correlation betweeen winter survival and snow mould injury (Table 5). Thus varieties with poor overwintering capacity were also susceptible to snow mould.

\section{Winter rye}

The winter survival rates of winter rye and triticale varieties at different locations in 1989-1992 are shown in Table 7. The analysis of variance indicated that in some trials the differences be- tween varieties were not significant (Table 7). In most cases this was due to a very mild winter (Mietoinen 1992, Jokioinen 1992 and Anjalankoski 1990).

The range of variation within winter rye trials is shown in Figure 2. The trials were separated into two groups based on the degree of variation. In group 1 there was extensive variation between the varieties, while in group 2 the variation was limited. There was in most cases a highly significant correlation between the results of trials within each group. In group 1 there was extensive variation between the varieties at Jokioinen 1991, Pälkäne 1990, 1991 and 1992, Anjalankoski 1992, Laukaa 1991 and Sotkamo 1991 and 1992. All these trials had a cold winter and prolonged snow cover. In trials at Jokioinen 1992, Mietoinen 1991 and 1992, Anjalankoski 1990 and Laukaa 1990 and 1992 there was only small variation between the rye varieties, and these trials made up group 2 . These trials were characterized by a rather mild winters. There was in most cases a high positive correlation between the results of group 1 trials, the total results of Finnish trials and the results of the Inter-Nordic trials, especially when the primary stress factor was snow (Table 2). In group 2 trials, the correlations with the Inter-Nordic trials and the Finnish totals 
Table 6. Snow mould (M.nivale) resistance of winter wheat, rye and triticale in 1990-1991 and 1991-1992 at different locations. A scoring system of 0 (no damage) to 10 (dead plant) was used. The differences between varieties were studied with the analysis of variance. ${ }^{*} \mathrm{p}<0.05,^{* *} \mathrm{p}<0.01$ and ${ }^{* * *} \mathrm{p}<0.001$.

Winter wheat

\begin{tabular}{|c|c|c|c|c|c|c|c|c|}
\hline \multirow[t]{2}{*}{ Variety } & \multirow{2}{*}{$\begin{array}{l}\text { Mietoinen (1) } \\
1990\end{array}$} & \multicolumn{2}{|c|}{ Jokioinen (2) } & \multicolumn{2}{|c|}{ Pălkăne (3) } & \multicolumn{2}{|c|}{ Laukaa (5) } & \multirow{2}{*}{$\begin{array}{c}\text { Sotkamo (6) } \\
1990\end{array}$} \\
\hline & & 1990 & 1991 & 1990 & 1991 & 1990 & 1991 & \\
\hline Linna & 0.25 & 0.75 & 1.25 & 1.50 & 5.75 & 2.00 & 1.50 & 2.00 \\
\hline Vakka & 0.75 & 1.25 & 1.00 & 2.25 & 8.25 & 1.75 & 2.00 & 5.00 \\
\hline Albidom & 2.00 & 1.00 & 1.50 & 2.00 & 6.50 & 2.00 & 3.00 & 5.00 \\
\hline Aura & 1.00 & 1.50 & 1.75 & 2.00 & 7.50 & 2.50 & 2.75 & 3.00 \\
\hline Kosack & 1.00 & 0.50 & 1.50 & 1.75 & 9.25 & 3.00 & 1.75 & 4.25 \\
\hline Walde & 1.00 & 2.50 & 1.75 & 3.25 & 8.75 & 1.50 & 2.00 & 3.25 \\
\hline Kharkow & 1.50 & 3.00 & 1.25 & 1.50 & 9.00 & 3.75 & 1.25 & 3.00 \\
\hline Miron. 808 & 0.75 & 3.25 & 1.00 & 3.25 & 9.25 & 3.25 & 1.75 & 4.50 \\
\hline Norstar & 1.25 & 0.75 & 1.50 & 3.25 & 8.75 & 5.00 & 3.25 & 5.50 \\
\hline Skjaldar & 1.75 & 2.50 & 0.50 & 3.00 & 6.25 & 4.25 & 1.25 & 5.25 \\
\hline Rida & 1.75 & 2.50 & 1.75 & 3.75 & 7.75 & 3.50 & 2.50 & 2.75 \\
\hline Folke & 1.25 & 1.25 & 1.25 & 0.50 & 7.75 & 2.75 & 1.00 & 2.50 \\
\hline Holme & 1.50 & 2.25 & 1.75 & 2.25 & 9.75 & 2.00 & 2.50 & 4.25 \\
\hline Hildur & 2.00 & 3.75 & 1.50 & 2.50 & 9.75 & 5.25 & 2.00 & 4.25 \\
\hline Kraka & 1.75 & 2.75 & 2.00 & 2.75 & 9.00 & 5.75 & 2.25 & 3.75 \\
\hline Sleipner & 1.25 & 1.00 & 1.25 & 2.25 & 3.25 & 5.00 & 2.00 & 2.25 \\
\hline Frederick & 2.00 & 5.00 & 2.00 & 3.67 & 9.50 & 3.50 & 4.50 & 5.50 \\
\hline Solid & 1.25 & 2.50 & 1.25 & 1.75 & 8.75 & 5.75 & 1.75 & 3.50 \\
\hline Goertzen & 1.50 & 4.25 & 1.50 & 3.25 & 9.75 & 7.75 & 2.50 & 6.50 \\
\hline Urban & 1.50 & 2.50 & 2.75 & 2.50 & 8.25 & 4.00 & 2.25 & 4.25 \\
\hline Gawain & 4.25 & 2.25 & 3.00 & 3.75 & 9.50 & 5.00 & 4.25 & 4.75 \\
\hline Apollo & 2.75 & 3.00 & 1.50 & 3.67 & 8.75 & 6.50 & 4.25 & 6.33 \\
\hline Longbow & 2.50 & 1.25 & 1.50 & 3.50 & 10.00 & 5.75 & 3.75 & 7.00 \\
\hline F-value & $3.05^{* * *}$ & $3.47^{* * *}$ & $2.10^{* *}$ & 1.81 & $8.62^{* * *}$ & 1.63 & $2.77^{* * *}$ & 0.86 \\
\hline
\end{tabular}

Rye and triticale

\begin{tabular}{|c|c|c|c|c|c|c|c|c|c|c|}
\hline \multirow[t]{2}{*}{ Variety } & \multirow{2}{*}{$\begin{array}{l}\text { Mietoinen (1) } \\
1990\end{array}$} & \multicolumn{2}{|c|}{ Jokioinen (2) } & \multicolumn{2}{|c|}{ Pälkäne (3) } & \multirow{2}{*}{$\begin{array}{c}\text { Anjala (4) } \\
1990\end{array}$} & \multicolumn{2}{|c|}{ Laukaa (5) } & \multicolumn{2}{|c|}{ Sotkamo (6) } \\
\hline & & 1990 & 1991 & 1990 & 1991 & & 1990 & 1991 & 1990 & 1991 \\
\hline Jussi & 0.50 & 4.25 & 1.75 & 3.00 & 1.25 & 0.25 & 4.25 & 1.50 & 1.00 & 0.00 \\
\hline Voima & 0.50 & 5.00 & 2.00 & 5.00 & 1.25 & 1.25 & 2.50 & 1.75 & 1.25 & 1.00 \\
\hline Anna & 0.75 & 5.25 & 2.00 & 4.25 & 1.50 & 0.75 & 3.00 & 2.25 & 2.50 & 1.50 \\
\hline Norderås tetra & 0.50 & 3.25 & 1.75 & 4.00 & 1.00 & 0.50 & 3.00 & 2.25 & 2.75 & 0.50 \\
\hline Vågonäs höstråg & 2.75 & 1.75 & 3.25 & 2.75 & 2.25 & 1.00 & 0.50 & 7.25 & 3.00 & 5.50 \\
\hline Talovskaja & 1.00 & 5.25 & 1.50 & 5.00 & 1.75 & 0.50 & 1.75 & 1.50 & 3.50 & 0.50 \\
\hline Musketeer & 3.00 & 7.50 & 1.00 & 4.67 & 6.00 & 1.50 & 1.75 & 3.25 & 1.75 & 0.00 \\
\hline Prima & 2.75 & 6.75 & 1.00 & 4.25 & 4.50 & 1.00 & 1.00 & 2.75 & 5.00 & 0.00 \\
\hline Dominator & 2.25 & 5.75 & 4.25 & 4.00 & 7.50 & 1.75 & 2.50 & 6.00 & 1.00 & 0.50 \\
\hline Kungs II & 2.50 & 7.25 & 4.75 & 3.25 & 7.00 & 0.75 & 3.50 & 5.50 & 3.25 & 1.50 \\
\hline Petkus II & 2.25 & 6.25 & 2.75 & 4.33 & 6.75 & 1.50 & 2.25 & 3.50 & 5.00 & 0.00 \\
\hline Epos & 2.00 & 5.50 & 3.50 & 3.67 & 7.00 & 0.75 & 3.50 & 3.25 & 2.75 & 1.00 \\
\hline Danko & 2.00 & 5.75 & 2.75 & 2.50 & 7.00 & 1.25 & 2.25 & 5.00 & 3.00 & 1.25 \\
\hline F-value & $7.24 * * *$ & $4.80^{* * *}$ & $2.71^{* *}$ & 0.99 & $27.06^{* * *}$ & 1.08 & $3.34^{* *}$ & $6.63^{* * *}$ & 1.63 & $6.30^{* * *}$ \\
\hline Sv 856003 & 1.50 & 1.75 & 1.25 & 1.25 & 8.75 & 3.75 & 1.50 & 2.25 & 1.50 & 0.50 \\
\hline Sj 868013 & 1.75 & 2.25 & 1.25 & - & 8.50 & 5.50 & 1.50 & 3.75 & 4.50 & 0.25 \\
\hline Dagro & 1.75 & 3.00 & 1.25 & - & 9.25 & 3.25 & 1.00 & 3.50 & 4.50 & 0.25 \\
\hline Uno & 2.50 & 2.50 & 1.00 & 0.50 & 9.00 & 3.40 & 0.00 & 2.75 & 4.50 & 1.50 \\
\hline Local & 2.75 & 7.50 & 1.75 & 2.25 & 8.75 & 7.75 & 0.25 & 7.25 & 6.25 & 1.50 \\
\hline F-value & 1.72 & $28.47^{* * *}$ & 0.30 & $4.27^{*}$ & 0.67 & 1.36 & $3.34^{*}$ & $8.28^{* * *}$ & 1.68 & $1.52^{* * *}$ \\
\hline
\end{tabular}




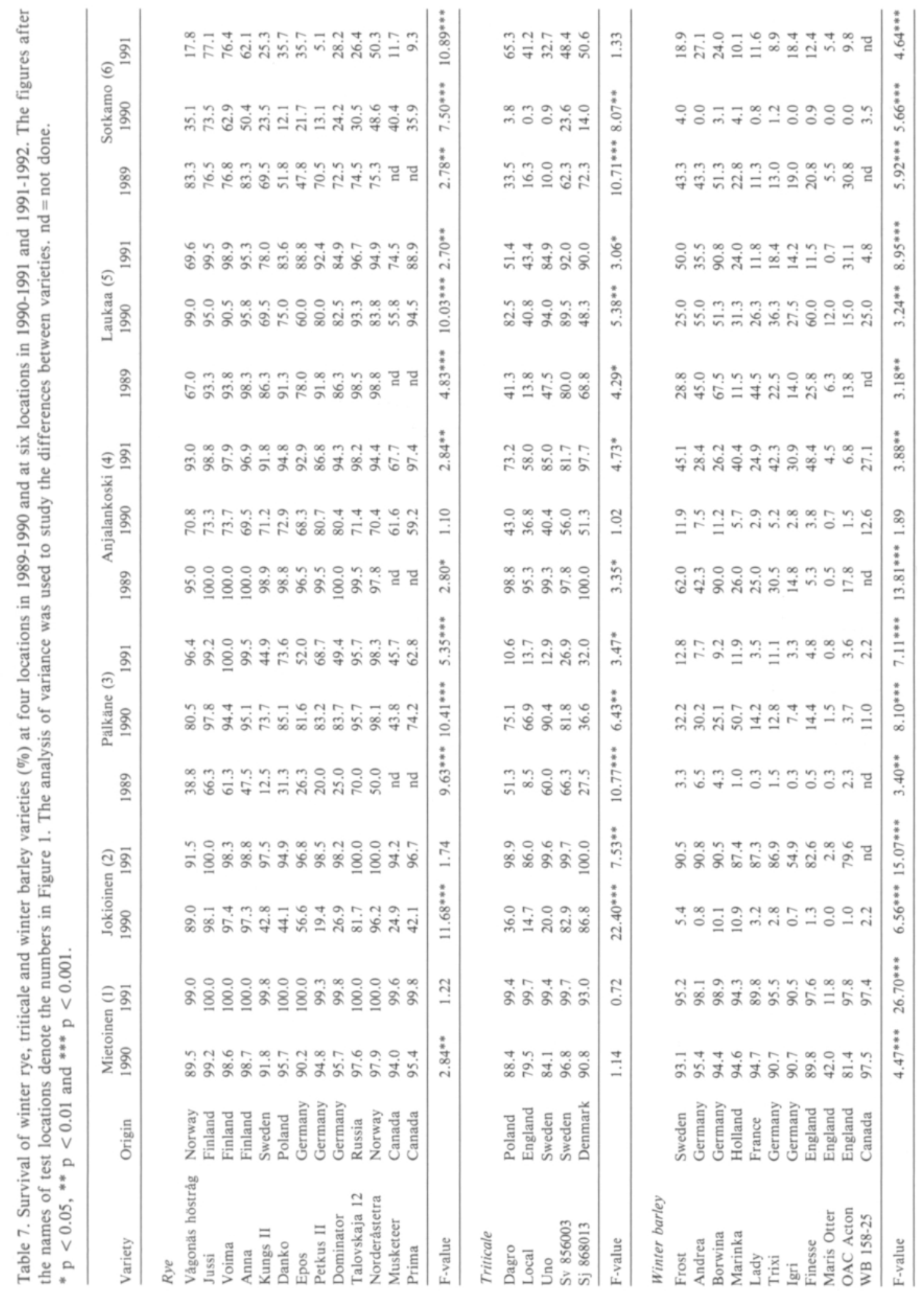


were mostly non significant. However, the results of the trials Jokioinen 1992, Mietoinen 1991 and Laukaa 1992 correlated positively with the totals of both the Inter-Nordic and the Finnish trials. The analysis of variance indicated that the differences between the experimental varieties were highly significant for both groups. The differences between the experimental sites and years were also highly significant during the test period (Table 3 ). The rye varieties were classified according to their winterhardiness within both groups on the basis of Tukey's Studentized Range test (Table 4). The classification in group 1 was quite analogous with the winterhardiness of varieties based on the total means of the Finnish trials. According to this grouping, the most winter hardy rye varieties were 'Jussi', 'Voima', 'Anna' and 'Norderåstetra' and those most susceptible were 'Petkus II' and 'Musketeer'. In group 2 with quite mild winters, the order of hardiness of the varieties was somewhat different from that of group 1. For example, 'Vågonäs höstråg' survived better cold and snowy winters than the mild ones. In the case of 'Petkus II', the situation was the opposite. Since the differences in winterhardiness of varieties were very small in group 2, the order of varieties in this group may be due to chance.

There was a positive correlation between winter survival and erect growth habit of winter rye varieties (Table 5). On average the growth habit of the rye varieties was more erect compared to the wheat varieties. At the end of the hardening period there was very little variation in the growth stage and height of plants among rye varieties. This was probably the reason for the lack of correlation between these factors and the winter survival of the varieties (Table 5). The correlation between growth stage and plant height and the snow mould damage to plants was also non significant, but negative as expected (Table 5).

The differences in snow mould ( $M$. nivale) damage among rye varieties were statistically significant in seven out of twelve trials (Table 6). There was a high negative correlation between the survival rate and snow mould injury in winter rye varieties (Table 5), indicating the importance of snow mould.

\section{Triticale}

The differences in the winter survival among triticale varieties were significant in seven out of 16 trials (Table 7). The ranges of variation within trials are shown in Figure 2. Although in some trials the winterhardiness of the most winter hardy triticale varieties was equal to that of the best winter wheat and rye varieties, the general winterhardiness of triticale varieties was similar to the medium or poorly winter hardy wheats (Fig. 2).

The results from trials with small variation in winter survival ability, either high survival (Jokioinen 1992, Mietoinen 1991 and 1992) or low survival (Pälkäne 1992, Anjalankoski 1991 and Sotkamo 1991 and 1992), correlated well with each other, and these trials made up group 1. Since the results from Laukaa 1990 correlated well with these results, they were also included in group 1.

There was also good correlation between trials with great variation in the survival abilities of varieties: Jokioinen 1991, Pälkäne 1990 and Laukaa 1991. These made up group 2.

The analysis of variance indicated that the differences in the survival rate among the varieties were highly significant in both groups (Table 3 ). The experimental sites, in group 1 also experimental years, differed from each other highly significantly, too. The most winter hardy triticale varieties were 'Sv 856003' and 'Sj 868013' and the least hardy was 'Local' (Table 4).

The correlations between the individual trials and Inter-Nordic trials could not be determined since triticale was excluded from the total InterNordic results.

The negative correlation between survival percentage and growth habit of triticale varieties indicates that prostrate growth habit might be beneficial for triticale as it was for winter wheat (Table 5). Positive but non significant correlations were observed between survival rate and growth stage and height of plants (Table 5).

There was a highly significant negative correlation between survival rate and snow mould resistance of triticale varieties (Table 5). The analysis of variance indicated that differences in snow mould resistance were significant only at Jokioinen 1991 
and at Laukaa 1992 (Table 6). The most snow mould resistant variety was 'Sv 856003' and the most susceptible was 'Local'.

\section{Winter barley}

The differences in the survival rates among winter barley varieties at different experimental sites in 1989-1992 are shown in Table 7. Three groups of winter barley trials could be formed based on the variation between varieties within trials (Fig. 2) and on the correlation between the results from different trials. Group 1 consisted of two trials (Pälkäne 1990 and Anjalankoski 1991), in which the survival percentage of all winter barley varieties was poor, and the variation between varieties was not significant (Table 7), and of five trials (Anjalankoski 1990, Laukaa 1990 and 1992 and Sotkamo 1990 and 1992) in which winter survival varied greatly with significant differences between varieties. In group 2 (Jokioinen 1991, Pälkäne 1991 and 1992 and Sotkamo 1991), the winter survival of varieties was poor, but the differences between the varieties were significant (Table 7). In group 3 (Jokioinen 1992 and Mietoinen 1991 and 1992), the survival rate of varieties was high, but there were significant differences in the winterhardiness between varieties.

There was a high positive correlation between the results of group 1 and group 3 trials and those of the Inter-Nordic trials (Table 2). The correlation between group 2 trials and Inter-Nordic trials was in some cases significant. In group 1 trials, most of the winters were cold with a prolonged snow cover. The group 3 trials were characterized by very mild winters, and most of the winter barley varieties survived very well. There were highly significant differences in the survival abilities of winter barley varieties within each of the groups (Table 3). The differences between the experimental sites were highly significant within each group, and in groups 1 and 2 the years differed from each other significantly, too. According to Tukey's Studentized Range test (Table 4), the most winter hardy barley varieties were 'Borwina', 'Andrea', 'Frost' and 'Marinka'. The most susceptible variety in all groups was 'Maris Otter'.
Growth habit and growth stage were not determined, since all the winter barley varieties were similar in this respect. The growth habit of all varieties was totally prostrate.

The snow mould resistance of winter barley varieties was not determined, because the stands were severely injured during the winter.

\section{Discussion}

The ability to survive under field conditions is considered to be the ultimate indication of a cultivar's winterhardiness potential. There are, however, biotic (snow moulds) and abiotic (frost, heaving and desiccation, ice encasement, flooding) stress factors, which may cause winter injury. Finland being situated between the maritime and continental climatic zones, has highly variable weather conditions during winter. All stress factors may occur during winter at the same site. It is therefore difficult to ascertain the major reason for winter injury. In addition, it is not possible to observe the sequence of events during the winter beneath a snow cover. For example, during a winter thaw, melted water percolates through the snow and builds up the frozen ground. These conditions may form ice encasement when the water refreezes. Alternate freezing and thawing in spring may cause the ice layer to disappear before the thawing. The weakened and damaged plant parts are then readily attacked by snow moulds. In such situations it is very difficult to determine the primary reason for winter injury. This was observed also in the present study especially in the case of winter wheat.

The winter survival of winter wheat, rye, triticale and winter barley varieties varied depending on the test location and the year. Rye was the most winter hardy species, winter wheat was somewhat hardier than triticale, although in some trials the survival of the most winter hardy triticale varieties was equal to that of the best winter wheat and rye varieties. Winter barley was the least winter hardy species. This is in accordance with the results of ANDREWS et al. (1986), ClOUtIER et al. (1990), FOWLER and CARLES (1979), FOWLER and LIMIN (1987), HÖMMÖ (1992) and KOLAR et al. (1991). Accord- 
ing to METCALF et al. (1970), the higher moisture content in winter barley crowns than in wheat, could affect the winter survival of the species. According to POMEROY et al. (1975), the reason for the lower overwintering potential of winter barley compared with winter wheat could be both the lower hardening and the lower rehardening capacity after repeated warm periods in winter.

There were significant differences in the weather conditions at different test locations during the test period. At the southern-most location, Mietoinen, the test winters were very mild, and there was some winter damage only to the least winter hardy winter wheat, triticale and winter barley cultivars.

In 1989-1990, trials were established only at four locations: Pälkäne, Anjalankoski, Laukaa and Sotkamo. At all locations the cold period was short, but the minimum air temperatures were low $\left(-18^{\circ} \mathrm{C}\right.$ $\left.-24^{\circ} \mathrm{C}\right)$. At Pälkäne, the ground was unfrozen at the time of the first snowfall, which created favourable conditions for snow mould infection. The great variation in the survival of winter rye and triticale varieties was obviously due to the damage caused by snow mould although it was not specified. At Anjalankoski, the winter was very mild, and only the winter barley varieties were damaged. At Laukaa and Sotkamo, the winter conditions favoured screening for winterhardiness. The cold period was long enough to cause damage to plants, and the snow cover gave protection against extreme temperatures.

The winter 1990-1991 was the most appropriate for screening cultivars under field conditions. The differences in winter survival among the varieties were significant at all locations but Mietoinen and Anjalankoski. The primary cause of damage to plants was, however, in most cases obscure. Snow mould occurred at all locations, but the differences between varieties for snow mould resistance were significant only at Jokioinen, Mietoinen (wheat, rye) and Laukaa (rye).

The winter 1991-1992 was quite mild at all test locations. The minimum temperatures were moderate and the continuous frost period was short, but the snow cover was deeper than average at Pälkäne and Laukaa. At Pälkäne, winter wheat and winter barley varieties were injured by ice. At Laukaa, ice formation did not occur, as the snow melted simultaneously with the warming up of the weather. However, the mild winter combined with the thick snow cover promoted intensive snow mould infection primarily to the rye and triticale varieties.

The individual trials, which produced similar results, were pooled to form larger entities for analysis of variance. In all species the differences between varieties within these groups were statistically very significant. The test locations and the years differed from each other also significantly. It is probable that in each trial different combinations of stress factors prevailed, and the varieties were ranked according to their tolerance to the dominant stress factor(s) in each trial. Thus the results of field trials always characterize rather the general winter survival ability of varieties than the resistance to some specific stress factor. Accordingly, STEPONKUS (1978) and VEISZ and RAJKI (1987) stated that the frost resistance of winter wheat cannot be evaluated under field conditions in countries where the weather in winter is variable, even in the case of trials performed under extreme conditions.

The genetic controll of winterhardiness is complicated, making the breeding of winter hardy cultivars for all different winter stress conditions difficult. According to BRULE-BABEL and FOWLER (1988) and SUTKA (1981), the coldhardiness of winter wheat is mainly controlled by additive gene action with possibly by some dominant genes. GULLORD (1974) divided frost resistance of winter wheat into high and low intensity freezing resistance, and stated that both are controlled by partially dominant genes which are mainly additive. FEDUlov et al. (1990) found also that the frost resistance of winter wheat is under polygenic control, but wheat varieties differ in their gene action, promoting frost resistance in the course of winter. Genetic control of coldhardiness is mainly additive also in rye (BRULE-BABEL and FOWLER 1989) and winter barley (EUNUS et al. 1962, ROHDE and PULHAM 1960).

According to BRUEHL (1982), the resistance to snow moulds is polygenic and non specific. ANDREWS and POMEROY (1975) and McKERSIE and HUNT (1987) found a close correlation between the frost tolerance and ice encasement tolerance of 
winter wheat varieties, but in both studies there were clear exceptions. A similar correlation was observed also in winter wheat by ANDREWS and GUDLEIFSSON (1983). According to GUDLEIFSSON and LARSEN (1993), winter rye has the highest ice encasement tolerance of the winter cereals followed by winter wheat and triticale, while winter barley was the least tolerant.

There was a very significant positive correlation between the results of most of the individual trials and the results of the Inter-Nordic trials, especially those with snow being the main stress factor. This was obviously due to the fact that the snow results were mostly based on the Finnish trials, whereas the frost results were collected from the other Nordic countries. But another explanation might be that the ranking of varieties is different under frost and snow stress conditions, and that in Finland breeding against snow conditions is more important.

The prostrate growth habit was found to be more beneficial for the winter survival of winter wheat and triticale, and the erect growth habit was more beneficial for rye. The erect winter wheat and triticale plants are probably easily damaged by e.g. frost, and the damaged plant parts are readily attacked by snow mould. Since the leaves of winter rye plants are more hardy, it is not so important for rye to grow under protective snow cover. On the other hand, the attack of snow mould is better avoided if the leaves grow upwards and not along the ground. These results are in accordance with the results of FOWLER and CARLES (1979), FOWLER et al. (1981) and ROBERTS (1990).

On the basis of the results in this study, the screening for general winterhardiness of cereals should be done in quite extreme conditions. Mietoinen and Jokioinen were best suited for the selection of winter barley, and Laukaa and Sotkamo for winter wheat, rye and triticale. The most winter hardy winter wheat varieties were 'Linna', 'Vakka', 'Kosack', 'Albidom 12' and 'Aura', the most hardy rye varieties 'Jussi', 'Voima', 'Anna' and 'Norderåstetra', and the most hardy triticales ' $\mathrm{Sv}$ 856003' and 'Sj 868013'. The most winter hardy barley varieties were 'Borwina', 'Andrea' and 'Frost'.

The winterhardiness of overwintering plants is, however, always a very complicated matter depending on the hardening conditions, e.g. temperature and light, and the prevailing stress factors during winter. In addition, the ranking of varieties on the basis of results from one location may be quite different from that of another location. For example, the winter wheat variety 'Linna', developed in Finland, is the most winter hardy winter wheat variety in Finland. However, when grown in eastern Ontario, Canada, the winterhardiness of this variety was only moderate (ANDREWS et al. 1986). Thus one should be very careful in adopting breeding materials and test methods based on local conditions.

Acknowledgements. Sincere thanks are due to Miss MarjaLeena Manninen, Miss Pia Kallio and Mr. Yrjö Karppinen for their technical assistance as well as to the people at the experimental stations who helped in the practical work, and Mrs. Sevastiana Ruusamo for correcting the English language. This study was supported financially by the SNP (Samnordic Planteforedling) and the Ministry of Agriculture and Forestry.

\section{References}

ANDERSEN, I. L. 1992. Winter injuries in grasslands in northern Norway caused by low temperature fungi. Norwegian J. Agric. Sci. 7 (Suppl.): 13-20.

ANDERSSON, S. 1986. Skördetidens och kvävegödslingens inverkan på vallens övervintring. NJF Seminar Nr. 84, Lantbruksväxtemas Övervintring. Jokioinen, Finland. p. $34-40$.

Andrews, C. J. \& Gudleifsson, B. E. 1983. A comparison of cold hardiness and ice encasement tolerance of timothy grass and winter wheat. Can. J. Plant Sci. 63: 429-435.
— \& Pomeroy, M. K. 1975. Survival and cold hardiness of winter wheats during partial and total ice immersion. Crop Sci. 15: 561-566.

—, Pomeroy, M. K. \& Seaman, W. L. 1986. The response of fallsown cereals to winter stresses in eastern Ontario. Can. J. Plant Sci. 66: 25-37.

Bengtsson, A. 1986. Såtider och benomylbehandling i höstråg och höstvete. NJF Seminar Nr. 84, Lantbruksväxternas Övervintring. Jokioinen, Finland. p. 4044. 
Brueht, G. W. 1982. Developing wheats resistant to snow mold in Washington State. Plant Disease 66: 1090-1095.

Brule-Babel, A. L. \& Fowler, D. B. 1987. Genetic control of cold hardiness in winter wheat. Can. J. Plant Sci. 67: 276.

— \& Fowler, D. B. 1988. Genetic control of cold hardiness and vernalization requirement in winter wheat. Crop Sci. 28: 879-884.

— \& Fowler, D. B. 1989. Genetic control of cold hardiness and vernalization requirement in rye. Genome. 32: 19-23.

Cloutier, Y., Comeau, A., Bernier-Cardou, M. \& Angers, D. A. 1990. Effect of soil water content on the winter survival of winter wheat, rye and triticale. Can. J. Plant Sci. 70: 667-675.

Dvorak, J. \& Fowler, D. B. 1978. Cold hardiness potential of triticale and tetraploid rye. Crop Sci. 18: 477-478.

Eunus, A. M., Johnson, L. P. V. \& AKsel, R. 1962. Inheritance of winterhardiness in an eighteen-parent diallel cross of barley. Can. J. Genet. Cytol. 4: 356-376.

Fedulov, Yu. P., Puchkov, Yu. M., Belikova, N. M. \& NaBAKov, G. D. 1990. Change in structure of frost resistance of winter wheat during wintering. Soviet Agricultural Sciences (USA) 6: 1-4.

Fowler, D. B. \& CARLEs, R. J. 1979. Growth, development, and cold tolerance of fall-acclimated cereal grains. Crop Sci. 19: 915-922.

—, Dvorak, J. \& Gusta, L. V. 1977. Comparative cold hardiness of several Triticum species and Secale cereale L.. Crop Sci. 17: 941-943.

— \& GuSTA, L. V. 1977. Influence of fall growth and development on cold tolerance of rye and wheat. Can. J. Plant Sci. 57: 751-755.

- , Gusta, L. V., Bowren, K. E., Crowle, W. L., Mal. Lough, E. D., McBean, D. S. \& McIver, R.N. 1976. Potential for winter wheat production in Saskatchewan. Can. J. Plant Sci. 56: 45-50.

— \& Limin, A. E. 1987. Exploitable genetic variability for cold tolerance in commercially grown cereals. Can. J. Plant Sci. 67: 278.

Gudleifsson, B. E. 1986. Ice encasement damages on grasses and winter cereals. NJF Seminar Nr. 84, Lantbruksväxternas Övervintring. Jokioinen, Finland. p. 5966.

— \& LARSEN, A. 1993. Ice encasement as a component of winter kill in herbage plants. In: Li, P.H. \& Christersson, L. (eds.). Advances in Plant Cold Hardiness. CRC Press, Boca Raton, Ann Arbor, London, Tokyo. p. 229-249.

Gullord, M. 1974. Genetics of freezing hardiness in winter wheat (Triticum aestivum L.). Dissertation for the degree of doctor of philosophy, Michigan State Univ. 70 p.

Gusta, L. V. 1986. The induction and maintenance of cold hardiness in winter cereals. NJF Seminar Nr. 84, Lantbruksväxternas Övervintring. Jokioinen, Finland. p. 928.

Hänninen, P. \& Jamalainen, E. A. 1968. Syysviljojen talvehtiminen Keski-Suomessa. Summary: Overwintering of winter cereals in Central Finland. Ann. Agric. Fenn. 7: 194-218.

Hensleigh, P. F., Blake, T. K. \& Welty, L. E. 1992. Natural selection on winter barley composite cross XXVI affects winter survival and associated traits. Crop Sci. 32: 57-62.

Hetherington, P. R., McKersie, B. D. \& Keeler, L. C. 1990. The influence of mineral nutrition on the expression of traits associated with winterhardiness of two winter wheat (Triticum aestivum L.) cultivars. Can. J. Plant Sci. 70: 443-454.

Номмӧ, L. M. 1992. Hardening ability of some winter wheat, winter rye and winter barley varieties. Use of conductivity method in evaluating the hardening ability of overwintering crop species. Norwegian J. Agric. Sci. 7 (Suppl.): 39-50.

Jamalainen, E. A. 1974. Resistance in winter cereals and grasses to low-temperature parasitic fungi. Ann. Rev. Phytopathol. 12: 281-302.

JEnkins, B. C. 1963. Secale additions and substitutions to common wheat. Proc. 2nd Int. Wheat Symp., Lund. Sweden. p. 301-310.

Kolar, S. C., Hayes, P. M., Chen, T. H. H. \& Linderman, R. G. 1991. Genotypic variation for cold tolerance in winter and facultative barley. Crop Sci. 31: 1149-1152.

Lazar, M. D., Chen, T. H. H., Gusta, L. V. \& Kartha, K. K. 1988. Somaclonal variation for freezing tolerance in a population derived from Norstar winter wheat. Theor. Appl. Genet. 75: 480-484.

LeVIrT, J. 1972. Responses of plants to environmental stresses. Physiological Ecology. Academic Press, New York. 697 p.

Limin, A. E., DvoraK, J. \& Fowler, D. B. 1985. Cold hardiness in hexaploid triticale. Can. J. Plant Sci. 65: 487-490.

— \& Fowler, D. B. 1984. The effect of cytoplasm on cold hardiness in alloplasmic rye (Secale cereale L.) and triticale. Can. J. Genet. Sytol. 26: 405-408.

— \& Fowler, D. B. 1986. Use of related species for the improvement of cold tolerance in wheat. Can. J. Plant Sci. 67: 282.

— \& Fowler, D. B. 1988. Cold hardiness expression in interspecific hybrids and amphiploids of the Triticae. Genome 30: 361-365.

— \& Fowler, D. B. 1991. Breeding for cold hardiness in winter wheat: problems, progress and alien gene expression. Field Crops Res. 27: 201-218.

McKersie, B. D. \& Hunt, L. A. 1987. Genotypic differences in tolerance of ice encasement, low temperature flooding, and freezing in winter wheat. Crop Sci. 27: 860-863.

Metcalf, E. L., Cress, C. E., Olein, C. R. \& Everson, E. H. 1970. Relationship between crown moisture content and killing temperature for three wheat and three barley cultivars. Crop Sci 10: 362-365.

NissınEN, O. 1986. Näringsämnens inverkan på utvintringssvamparnas angrepsgrad. NJF Seminar Nr. 84, Lantbruksväxternas Övervintring. Jokioinen, Finland. p. 101-107.

Olvång, H. 1992. Chemical control of winter damaging fungi in cereals. Norwegian J. Agric. Sci. 7(Suppl.): 5563.

Pohjakallio, 0., Salonen, A., Antilla, S. \& Halkil.ahti A.-M. 1962. Syysviljan talvehtimiseen vaikuttavista tekijöistä. Summary: Studies on factors affecting the over- 
wintering of winter cereals. Maatalous ja Koetoiminta 16: 140-152.

Pomeroy, M. K. \& Andrews, C. J. 1989. Low temperature injury in winter cereals. In: Li, P.H. (ed.). Low Temperature Stress Physiology in Crops. CRC Press, Boca Raton, FL. p. 107-122.

-, Andrews, C. J. \& FedaK, G. 1975. Cold hardening and dehardening responses in winter wheat and winter barley. Can. J. Plant Sci. 55: 529-535.

Pulı, S. 1986. Climatic factors in relation to winterhardiness. NJF Seminar Nr. 84, Lantbruksväxternas Övervintring. Jokioinen, Finland. p. 48-59.

ROBERTS, D. W. A. 1990. Identification of loci on chromosome $5 \mathrm{~A}$ of wheat involved in control of cold hardiness, vernalization, leaf length, rosette growth habit, and height of hardened plants. Genome 33: 247-259.

Rohde, C. R. \& Pulham, C. F. 1960. Heritability estimates of winter hardiness in winter barley determined by the standard unit method of regression analysis. Agron. J. 52: 584-586.

Sмrth, J. D. 1986. Snow mould diseases of crop plants in western Canada. NJF Seminar Nr. 84, Lantbruksväxternas Övervintring. Jokioinen, Finland. p. 119-129.
- 1987. Winter-hardiness and overwintering diseases of amenity turfgrasses with special reference to the Canadian Prairies. Agric. Can. Res. Branch Techn. Bull. 198712E. 192 p.

STEPONKUS, P. L. 1978. Cold hardiness and freezing injury of agronomic crops. Adv. Agr. 30: 51-98.

SUTKA, J. 1981. Genetic studies of frost resistance in wheat. Theor. Appl. Genet. 59: 145-152.

URVAS, L. 1986. The effect of fertilization on the overwintering of timothy. NJF Seminar Nr. 84, Lantbruksväxternas Övervintring. Jokioinen, Finland. p. 29-34.

VeISZ, O. \& RAJKI, E. 1987. Frost resistance in various winter wheat varieties during winter. Acta Agronomica Hungarica 36: 285-294.

\section{Manuscript received July 1993}

Leena Hömmö

Seppo Pulli

Agricultural Research Centre of Finland

Institute of Plant Breeding

FIN-31600 Jokioinen, Finland

\title{
SELOSTUS
}

\section{Syysvehnä-(Triticum aestivum), syysruis-(Secale cereale), ruisvehnä-( $\times$ Triticosecale $)$ ja syysohra (Hordeum vulgare) lajikkeiden talvenkestävyys kuudella koepaikalla Suomessa}

\author{
LEENA HÖMMÖ ja SEPPO PULLI
}

Maatalouden tutkimuskeskus

Tutkimuksessa selvitettiin kolmen vuoden (1989-1992) aikana 24 syysvehnä-, 13 ruis-, 5 ruisvehnä- (triticale) ja 11 syysohralajikkeen talvenkestävyyttä kuudella eri koepaikalla (Mietoinen, Jokioinen, Pälkäne, Anjalankoski, Laukaa ja Sotkamo). Lajikkeet oli valittu eri puolilta syysviljojen viljelyvyöhykettä, ja niiden talvenkestävyydessä tiedettiin olevan suurta vaihtelua hyvin kestävistä huonosti talvehtiviin, lähes kevätmuotoja muistuttaviin lajikkeisiin.

Kokeen tulosten perusteella ruis on syysviljoista selvästi talvenkestävin. Syysvehnä ja ruisvehnä ovat talvenkestävyydeltään hyvin lähellä toisiaan ja huomattavasti syysohraa kestävämpiä. Lajikkeiden talvenkestävyys oli tilastollisesti erittäin merkitsevästi erilainen kaikissa niissä kokeissa, joiden aikana oli ollut kylmä ja runsasluminen talvi. Talvet olivat koejakson aikana hyvin vaihtelevia, ja eri koepaikoilla kasvit joutuivat selviytymään hyvin erilaisista stressitekijöistä. Kasvien yleisen talvenkestävyyden katsotaan olevan additiivisen, osittain dominanssiin perustuvan geenitoiminnan säätelemää, jolloin erilaisissa stressiolosuhteissa lajikkeiden kestävyysjärjestys on aina hieman erilainen.
Kokeiden perusteella syysohran talvenkestävyyden testaamiseen soveltuu koepaikoista parhaiten Mietoinen ja Jokioinen, kun taas syysvehnän, rukiin ja ruisvehnän lajike-erot tulivat parhaiten esille Laukaassa ja Sotkamossa. Kestävimmät syysvehnälajikkeet Suomen oloissa olivat: Linna, Vakka, Kosack, Albidom 12 ja Aura. Ruislajikkeista parhaiten talvehtivat: Jussi, Voima, Anna ja Norderås tetra ja ruisvehnistä linjat Sv 856003 ja Sj 868013.

Syysohraa ei Suomessa viljellä kaupallisesti, mutta kestävimpien lajikkeiden: Borwina, Andrea ja Frost talvenkestävyys näyttäisi riittävän viljelyyn eteläisessä ja Lounais-Suomessa.

Syysvehnän ja ruisvehnän talvehtimiselle näyttää kokeen tulosten perusteella olevan edullista matala kasvutapa, kun taas ruis talvehtii paremmin, jos se on kasvutavaltaan pysty.

Kokeiden tulokset korreloivat yleensä erittäin hyvin yhteispohjoismaisten, vastaavien kenttäkokeiden kanssa. Erityisen hyvä korrelaatio oli niiden kokeiden kanssa, joissa runsas lumi oli katsottu tärkeimmäksi stressitekijäksi. 